\title{
Manifestaciones bucales de enfermedades de transmisión sexual identificadas en tres servicios de estomatología en Sur América
}

\author{
Oral manifestations of sexually transmitted diseases identified in three stomatology services in South America
}

\author{
Martha Carmona-Lorduy' orcid.org/0000-0003-3066-2219 \\ Iván Porto-Puerta ${ }^{2 *}$ orcid.org/0000-0002-5181-9855 \\ Héctor Lanfranch ${ }^{2}$ orcid.org/0000-0002-4596-2671 \\ Wanda Medina-Carmona ${ }^{1}$ orcid.org/0000-0002-6765-1188 \\ Laura Werner2,3 orcid.org/0000-0002-3826-4672 \\ Stella Maturana² orcid.org/0000-0002-6715-0332
}

1 Grupo de Investigación GITOUC, Facultad de Odontología, Universidad de Cartagena. Cartagena, Colombia

2 Facultad de Odontología, Universidad de Buenos Aires. Buenos Aires, Argentina

3 Servicio de Estomatología, Hospital Alemán Buenos Aires. Buenos Aires, Argentina

\section{Resumen}

Introducción: Las enfermedades de transmisión sexual se definen como un grupo de infecciones causadas por diversos agentes que se adquieren durante las relaciones sexuales. Éstas además suelen generar manifestaciones en boca. Objetivo: Determinar las lesiones características en cavidad bucal de enfermedades de transmisión sexual. Materiales y métodos: Estudio descriptivo transversal, con 37 pacientes que asistieron a los servicios de Estomatología de la Universidad de Buenos Aires, Universidad de Cartagena y el Hospital Alemán en Buenos Aires durante 2015 y 2016. Se realizó historia clínica completa con pruebas Venereal Disease Research Laboratory (VDRL) y Fluorescent Treponemal Antibody Absortion (FTA-ABS) en pacientes con presunción de sífilis. Además de análisis histopatológico y Reacción en Cadena de la Polimerasa (PCR) en pacientes con presunción de Virus de Papiloma Humano (VPH). Resultados: El promedio de edad de los pacientes fue de 38 años, predominó el sexo masculino. El $54,1 \%$ fueron diagnosticados con sífilis y la lesión más encontrada en estos fue la pápula. El 45,9\% restante fueron diagnosticados con VPH, la lesión predominante en estos fue la verrugosidad. Conclusiones: El odontólogo debe contribuir en la detección temprana de enfermedades de transmisión sexual identificando manifestaciones de éstas en boca; con el fin de evitar su evolución y prevenir su transmisión.

Palabras clave: Infecciones por papillomavirus; sífilis; serodiagnóstico de la sífilis; chancro; enfermedades de transmisión sexual; medicina oral; medicina interna. (Fuente: DeCS, Bireme).

\begin{abstract}
Introduction: Sexually transmitted diseases are defined as a group of infections caused by various agents which are acquired during sexual intercourse. They also tend to generate manifestations in the mouth. Objective: To determine the typical lesions in oral cavity of sexually transmitted diseases. Materials and methods: A descriptive transversal study was conducted with 37 patients who attended the stomatology services of the University of Buenos Aires, University of Cartagena and the Aleman Hospital in Buenos Aires during 2015 and 2016. A complete clinical history was carried out with Venereal Disease Research Laboratory (VDRL) and Fluorescent Treponemal Antibody Absortion
\end{abstract}


(FTA-ABS) tests in patients with presumption of syphilis. In addition, histopathological analysis and Polymerase Chain Reaction (PCR) was made in patients with presumption of Human papillomavirus (HPV). Results: The average age of the patients was 38 , where male sex predominated. $54.1 \%$ were diagnosed with syphilis and the most found lesion in them was the papule. The remaining $45.9 \%$ were diagnosed with HPV, the predominant lesion in them was a wart. Conclusions: The dentist should contribute to the early detection of sexually transmitted diseases by identifying manifestations of these in the mouth in order to prevent their evolution and prevent their transmission.

Keywords: Papillomavirus infections; syphilis; syphilis serodiagnosis; chancre; sexually transmitted diseases; oral medicine; internal medicine. (Source: DeCS, Bireme).

\section{Introducción}

Las enfermedades de transmisión sexual (ETS), alguna vez llamadas enfermedades venéreas, se definen como un grupo de patologías causadas por diversos agentes infecciosos como bacterias, hongos y virus que se adquieren durante la actividad sexual(1). El incremento de la prevalencia en estas enfermedades va de la mano de diferentes factores que han contribuido a su ascenso, entre los que se destacan cambios en el comportamiento sexual y social debido a la urbanización, industrialización, facilidad de viajar, cambios en la mentalidad referente a la virginidad, la tendencia actual a una mayor precocidad y promiscuidad sexual, acompañado de patrones de machismo que imperan en algunos países del mundo(2).

Según el Centers for Disease Control and Prevention (CDC) en el 2008 en Estados Unidos se diagnosticaron 20 millones de infecciones de transmisión sexual. La infección por VPH fue la más común llegando al $49 \%$ y la sífilis al $20 \%$. Las edades más afectadas en esta región son de 15-24 años(3). Todas estas infecciones de transmisión sexual suelen tener manifestaciones en cavidad bucal, por lo tanto se considera necesario que el odontólogo tenga amplio conocimiento de estas, para así identificar y diagnosticar a tiempo dichas patologías previniendo su contagio y progresión.

La sífilis es una enfermedad infecciosa con afección sistémica causada por la bacteria Treponema pallidum, perteneciente al orden Spirochaetales, familia Spirochaetaceae. Son organismos de diámetro exiguo, con morfología característicamente enrollada, presentan un movimiento rotatorio y ondulado sobre su eje central(4). La infección se adquiere principalmente por contacto sexual; también de forma congénita a través de la placenta, por transfusión de sangre humana contaminada o por inoculación accidental $\operatorname{directa}^{(5)}$.

La historia de natural de la sífilis se desarrolla en tres fases: primaria, secundaria y terciaria; cada una de estas genera afecciones sistémicas y en cavidad bucal. Las manifestaciones de la fase primaria aparecen entre los 10 días hasta los 2 meses después del contacto, se caracteriza por la aparición del chancro sifilítico en el lugar de la inoculación; esta lesión es una úlcera, de bordes indurados, redondeada de color rosa pálido. Si no se realiza tratamiento en un promedio de tres semanas, aparecen pápulas y lesiones queratósicas y ulcerativas en manos, pies y cavidad bucal, especialmente en bordes linguales, correspondientes a sífilis secundaria(6).

En su etapa tardía o terciaria algunas veces no se genera ningún tipo de manifestación, sin embargo cuando se presenta la goma sifilítica causa una perforación en paladar duro que comunica la cavidad nasal con la oral. En este estado es muy común la transmisión vertical entre madre e hijo, causando al recién nacido diversas malformaciones; muchas veces mortales ${ }^{(7)}$.

Para el diagnóstico de la sífilis, existen diferentes tipos de pruebas de laboratorios clasificadas en dos grupos: las pruebas no treponémicas tales como: la Venereal Disease Research Laboratory (VDRL), Reagina Plasmática Rápida (RPR), Enzyme-linked Inmunosorbent Assay (ELISA) y Unheated Serum Reagin (USR), por otro lado está el grupo de las pruebas treponémicas las cuales se utilizan para confirmar los resultados positivos obtenidos con las pruebas reagínicas, estas son: la Fluorescent-Treponemal antibody absorbed (FTAABS), la Treponema pallidum, Haemagglutination Assay (TPHA) y Western Blot(8). 
Para el tratamiento de dicha patología se debe aplicar antibioticoterapia lo más pronto posible con Penicilina G benzatínica en dosis de 2,4 millones de unidades inyectadas por vía intramuscular en una sola sesión; en pacientes alérgicos a la penicilina en la fase primaria y secundaria se recomienda Doxiciclina vía oral 100 mg por 21 días y en fase tardía o terciaria es recomendable desensibilización para tratar con Penicilina ${ }^{(9)}$.

Otra enfermedad de transmisión sexual es la infección por Virus de Papiloma Humano (VPH), este virus pertenece a la familia Papilomaviridae e infecta y se replica en el núcleo de células epiteliales. Posee una estructura relativamente simple: una cápside proteica de simetría icosaédrica y en su interior el material genético bajo la forma de ADN de doble cadena circular y carece de envoltura, por lo cual se denomina virus desnudo(10,11).

Los tipos de VPH considerados de alto riesgo de generar lesiones malignas, se asocian prácticamente al $100 \%$ de los casos de cáncer de cuello uterino; también se vinculan con el desarrollo de neoplasias extracervicales, tales como el cáncere de vagina, pene, ano, cabeza y cuello, en especial la orofaringe (cavidad nasal y cavidad bucal). Sin embargo, la incidencia de todos los cánceres extracervicales asociados al VPH es de sólo el 12\% del total de los cánceres producidos por este virus(12). Los tipos de VPH de bajo riesgo son tipos $6,11,42,43$ y 44, los cuales presentan un mínimo riesgo de progresión maligna, y los subtipos de alto riesgo son 16,18 , $31,33,35,39,45,51,52,56,58$ y 59 , que bajo la forma de infección persistente, pueden conducir a la transformación neoplásica(13).

La Reacción en Cadena de la Polimerasa (PCR) tanto convencional como en tiempo real y/o la hibridación in situ de ácidos nucleicos son las pruebas indicadas para la detección de los serotipos virales, ya que el estudio anatomopatológico que se realiza posterior a una biopsia, sólo muestra alteraciones celulares en el estrato epidérmico como coilocitosis, papilomatosis y acantosis que indican la presencia viral(14).
Acerca de las lesiones características reportadas en cavidad bucal se encuentran las lesiones verrugosas, vegetativas y pápulas, generalmente asintomáticas, relacionadas con prácticas sexuales oro-genitales. El condiloma acuminado es el tipo de lesión más frecuentemente reportado. Los tipos más relacionados con lesiones bucales son los 16, 18, 13 y 32, estos 2 últimos, asociado a la hiperplasia epitelial focal (Enfermedad de Heck)(15). El tratamiento para dichas lesiones depende de la severidad y progresión de la infección. En las mucosas, se recomienda la extirpación quirúrgica, y la aplicación de ácido tricloroacético; que ha demostrado grandes resultados sin recidiva en pacientes con hiperplasia epitelial focal(16).

El objetivo principal de este estudio fue determinar las lesiones características en cavidad bucal de pacientes con enfermedades de transmisión sexual y describir características epidemiológicas de dicha población.

\section{Materiales y métodos}

Se realizó un estudio descriptivo de corte transversal donde participaron 37 pacientes que acudieron a los servicios de Estomatología de las Facultades de Odontología de la Universidad de Buenos Aires, Universidad de Cartagena y del Hospital Alemán en Buenos Aires entre los años 2015 y 2016, esta muestra se obtuvo a partir de muestreo por conveniencia (no probabilístico), donde se incluyeron pacientes que consultaron dichos servicios con presunción de sífilis e infección por VPH; se excluyeron los pacientes que consultaban con diagnóstico presuntivo de otro tipo de infección de transmisión sexual, no hubo exclusión por sexo, edad, estrato socioeconómico, nacionalidad, género, ni religión.

Al total de pacientes se les realizó una historia clínica exhaustiva, incluyendo examen estomatológico minucioso con pruebas de VDRL y FTA-ABS en pacientes con presunción de sífilis; análisis histopatológicos y PCR en pacientes con presunción de VPH realizada con partidores dirigidos al gen tpN47(17). Estas se consideran pruebas confirmatorias y confiables para el 
diagnóstico serológico y molecular de estas enfermedades.

\section{Análisis estadístico}

Luego de la obtención de datos consignados en la historia clínica semiológica, fotografías clínicas intra y extraorales, pruebas serológicas, estudio anatomopatológico, y PCR se procedió a la organización estructuración y análisis de estos. Se realizaron pruebas de estadística descriptiva tales como frecuencia, porcentaje y promedio, utilizando el software SPSS (Statistical Package for the Social Sciences) licenciado.

\section{Consideraciones éticas}

Todos los pacientes que participaron en este estudio dejaron constancia de su aceptación para entrar en la investigación, la divulgación de resultados obtenidos así como de fotografías, firmando un consentimiento informado, en el cual se explica que es un estudio con fines académicos y que no se divulgaría su identidad. Esta investigación se considera de riesgo mínimo según la Resolución 8430 de 1993, Artículo Nº 11. Contó con la aprobación de los Comités de Ética de la Universidad de Cartagena y de la Universidad de Buenos Aires.

\section{Resultados}

De los 37 pacientes el 54,1\% (20) corresponden al sexo masculino y el $45,9 \%$ (17) al femenino. El promedio de edad fue de 38 años en un rango de 16 años a 72 años. Cuando se analizaron los hábitos, el 32\% de ellos eran fumadores, el 8,1\% eran consumidores de alcohol frecuente y el $59,9 \%$ no presentaba estos hábitos.

De los 37 pacientes, $54,1 \%$ (20) fueron diagnosticados con sífilis; 51,35\% (19) presentaba su forma secundaria y solo $2,70 \%$ (1), la forma primaria. No se encontró la enfermedad en su forma terciaria. 45,9\% (17) del total de la muestra fueron diagnosticados con infección por el $\mathrm{VPH}, 27,02 \%$ (10) estaban infectados por el serotipo $11,13,51 \%$ (5) por el serotipo 6 y $5,40 \%$ (2) por el serotipo 9 (Tabla 1).
Tabla 1. Distribución de las ETS de acuerdo a las formas presentadas

\begin{tabular}{llrr}
\hline \multicolumn{1}{c}{ ETS } & \multicolumn{1}{c}{ Subtipo } & $\boldsymbol{n}$ & \multicolumn{1}{c}{$\%$} \\
\hline Sífilis & Secundaria & 19 & 51,4 \\
& Primaria & 1 & 2,7 \\
& Terciaria & 0 & 0 \\
Virus del & Tipo 11 & 10 & 27 \\
papiloma & Tipo 6 & 5 & 13,51 \\
humano & Tipo 9 & 2 & 5,41 \\
Total & & $\mathbf{3 7}$ & $\mathbf{1 0 0}$ \\
\hline
\end{tabular}

Al analizar las lesiones elementales de los pacientes con sífilis primaria se encontró principalmente la úlcera (Figura 1). Y en los pacientes con sífilis secundaria, la lesión más frecuente fue la pápula $(57,8 \%)$, seguida por la úlcera $(36,8 \%)$ y la queratosis $(5,2 \%)$. (Figura 2$)$, (Tabla 2).

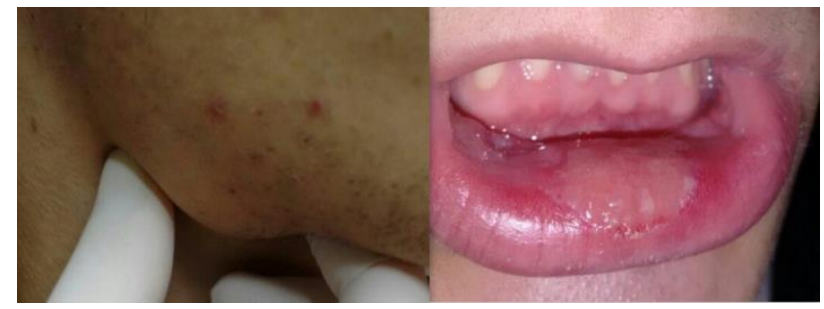

Figura 1. Ganglio satélite submandibular y chancro sifilítico, manifestaciones clave para el diagnóstico de sífilis primaria

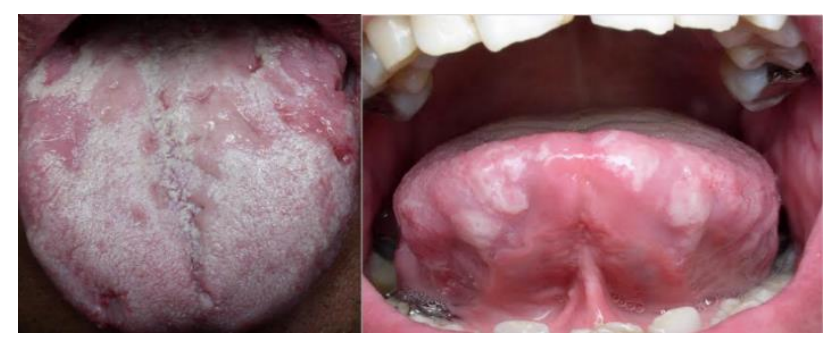

Figura 2. Úlceras y pápulas en caras dorsal y ventral de lengua, lesiones características de sífilis secundaria

Tabla 2. Distribución de lesiones elementales encontradas en los pacientes con sífilis secundaria

\begin{tabular}{lrr}
\hline $\begin{array}{l}\text { Tipo de lesión de } \\
\text { sífilis secundaria }\end{array}$ & $\boldsymbol{n}$ & \multicolumn{1}{c}{$\%$} \\
\hline Pápula & 11 & 57,9 \\
Úlcera & 7 & 36,8 \\
Queratosis & 1 & 5,3 \\
Total & $\mathbf{1 9}$ & $\mathbf{1 0 0}$ \\
\hline
\end{tabular}


En los 17 pacientes diagnosticados con infección del VPH se encontró que la lesión elemental más frecuente fue la verrugosidad (70,5\%), luego la vegetación $(23,5 \%)$ y manchas y placas $(5,8 \%)$. (Tabla 3) (Figura 3).

Tabla 3. Descripción de lesiones elementales encontradas en pacientes con infección por VPH

\begin{tabular}{lrr}
\hline $\begin{array}{c}\text { Tipo de lesión asociada } \\
\text { a infección por VPH }\end{array}$ & $\boldsymbol{n}$ & \multicolumn{1}{c}{$\%$} \\
\hline Verrugosidad & 12 & 70,6 \\
Vegetación & 4 & 23,5 \\
Manchas y placas & 1 & 5,9 \\
Total & $\mathbf{1 7}$ & $\mathbf{1 0 0}$ \\
\hline
\end{tabular}

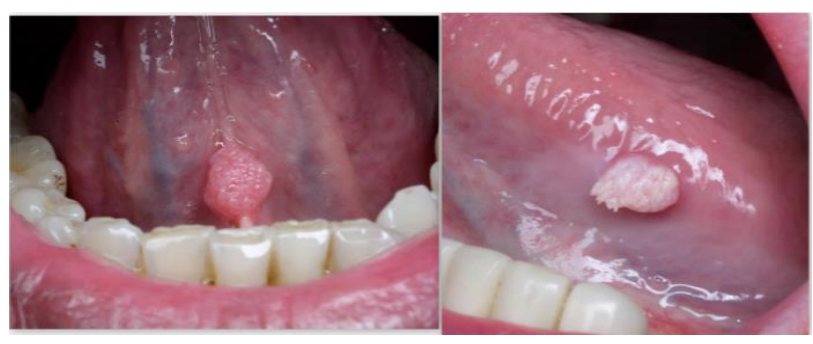

Figura 3. Vegetación y verrugosidad, manifestaciones características de infección por VPH

Las estructuras anatómicas en cavidad bucal más afectadas, en pacientes diagnosticados con sífilis o VPH fueron: el paladar blando en el 32,4\% (12); seguida de la cara dorsal de la lengua en $29,7 \%$ (11) semimucosa labial en $24,3 \%$ (9); en la mucosa yugal en 21,6\% (8); los bordes de la lengua en $18,9 \%$ (7), encías en $16,2 \%$ (6) y por último la cara ventral de lengua en 13,5\% (5). (Tabla 4).

Tabla 4. Descripción de estructuras anatómicas más afectadas por lesiones de sífilis o VPH

\begin{tabular}{|c|c|c|c|c|}
\hline \multirow[t]{2}{*}{ Sitio de presentación } & \multirow[t]{2}{*}{$\mathbf{n}$} & \multirow[t]{2}{*}{$\%$} & \multicolumn{2}{|c|}{$\begin{array}{l}\text { Distribución por } \\
\text { patologías (n) }\end{array}$} \\
\hline & & & VPH & Sífilis \\
\hline Paladar blando & 12 & 32,4 & 9 & 3 \\
\hline Cara dorsal de lengua & 11 & 29,7 & 7 & 4 \\
\hline Semimucosa labial & 9 & 24,3 & 7 & 2 \\
\hline Mucosa yugal & 8 & 21,6 & 5 & 3 \\
\hline $\begin{array}{l}\text { Bordes laterales de } \\
\text { lengua }\end{array}$ & 7 & 18,9 & 6 & 1 \\
\hline Encía & 6 & 16,2 & 4 & 2 \\
\hline Cara ventral de lengua & 5 & 13,5 & 5 & 0 \\
\hline
\end{tabular}

Del total de pacientes sólo 10,8\% (4) eran VIH positivo, de ellos 50\% (2) presentaba VPH tipo 6 y el otro $50 \%$ sífilis secundaria.

\section{Discusión}

Debido a que la literatura actual es escasa, no es posible recopilar estudios, sin embargo es posible comparar la presente investigación con reportes de caso recientes, en los cuales se describen las lesiones características de las patologías estudiadas (17-28).

Aguayo et al.(17), reportan un caso de sífilis en su forma primaria; la manifestación principal fue un nódulo de consistencia dura, asintomático, con superficie erosionada cubierta de costra serosa, localizado en el labio superior; en la anamnesis el paciente reconoció haber realizado prácticas sexuales orales; para su diagnóstico se realizaron pruebas de VDRL y biopsia excinsional que resultaron positivas a sífilis primaria. Este estudio no se asemeja a lo planteado en el presente manuscrito, primero que todo por el tipo de la lesión reportada, debido a que el nódulo no se encontró en la población estudiada, por otro lado, realizó VDRL y biopsia para diagnosticar; mientras que en el presente estudio se usó FTAABS y sólo como confirmatoria VDRL, este método diagnóstico coincide con diversas publicaciones(18-19).

Castro et al.(20), describen un caso clínico sobre sífilis secundaria, el paciente presentó lesiones placo-ulcerativas dolorosas que involucraban paladar blando, labio superior e inferior, además placas eritematosas en las palmas de las manos; se realizó prueba de VDRL que resulta positiva. Con dicho reporte se discrepa, debido a que las lesiones bucales reportadas en este estudio se encuentran en muy bajo porcentaje. Sin embargo, coincide con la utilización del análisis de VDRL como prueba diagnóstica.

Chairez et al.(21), plantean que las lesiones más encontradas en las infecciones por VPH son la verrugas $\mathrm{y}$ vegetaciones relacionadas con condiloma acuminado y papiloma bucal ubicadas en el paladar, concordando con este estudio, pues 
la verrugosidad fue la lesión elemental más encontrada y el paladar el sitio más afectado.

Asimismo, Delgado et al.(22), reportaron un caso de infección por VPH y las lesiones con vegetaciones de color violeta y blanco en caras dorsal y ventral de lengua, se realizó PCR e hibridación in situ de ácidos nucleicos para el diagnóstico de condiloma acuminado, coincidiendo con lo descrito en el presente manuscrito, donde se encontró gran variedad de este tipo de lesiones, y el uso de las mismas pruebas para el diagnóstico y tipificación viral.

En este estudio se presentan cuatro pacientes VIH positivo, que se considera un porcentaje bajo si se compara con otros reportes similares(23-24). Se razona que esta discrepancia se da debido a la antigüedad de más de 11 años de dichos estudios $^{(24,25)}$. Diversos estudios reportan un porcentaje de hasta un $70 \%$ de pacientes con VIH los cuales presentan sífilis o VPH(25,26). Estudios en este tipo de pacientes se consideran pertinentes debido a que la sífilis tiende a progresar en etapas tempranas a neurosífilis y el VPH se manifiesta de forma más rápida y agresiva en pacientes VIH positivo, por ende su prevención es crucial(27,28).

\section{Conclusiones}

Esta investigación es un punto de partida para futuros estudios acerca de enfermedades de transmisión sexual por cuanto no existen publicaciones de registros epidemiológicos suramericanos que indiquen el comportamiento bucal de dichas patologías. Se considera pertinente realizar mayores investigaciones en este campo y la divulgación de estas, con el fin de capacitar a odontólogos y especialistas en la identificación de dichas manifestaciones bucales y de esta manera prevenir la evolución y transmisión de las mencionadas enfermedades.

\section{Conflicto de intereses}

Ninguno declarado por los autores.

\section{Referencias}

1. Álvarez M, De la torre L, Domínguez J. Las Infecciones de Transmisión Sexual: una revisión dirigida a la atención primaria de salud. Revista Cubana de Medicina General Integral. 2014;30(3):343-353.

2. Cortés A, Sordo M, Cumbá C, García R, Fuentes J. Comportamiento sexual y enfermedades de transmisión sexual en adolescentes de secundaria básica de Ciudad de la Habana 1996. Revista Cubana de Higiene y Epidemiologia. 2000;38(1):53-59.

3. Satterwhite C. Sexually transmitted infections among U.S. women and men: Prevalence and incidence estimates, 2008. Sex Transm Dis. 2013;40(3):187-193.

4. Diez M.; Díaz A. Infecciones de transmisión sexual: epidemiología y control. Revista española de Sanidad Penitenciaria. 2011;13(2):58-66.

5. Lopez J, Frasquet J. Sífilis: una revisión actual. Madrid: Centro de Control de Calidad SEIMC; 2011.

6. Nieto, T, Larrea, S, Moreno, G, Pinto, M, Iturralde P. Infecciones de transmisión sexual, prevalencia de VIH/SIDA sífilis, en estudiantes adolescentes de 5 y 6 tos cursos, encuesta de conocimientos, actitudes y practicas (CAP) en el Cantón Ibarra. Revista Científica y Tecnológica "El Investigador". 2012(4):60-69.

7. Lugones M, Molinet I, Quintana T, Vázquez M. Sífilis y gonorrea; parte de su historia. Revista Cubana de Medicina General Integral. 1995;11(4):382-384.

8. Berdasquera D, Lazo M, Galindo B, Gala A. Sífilis: pasado y presente. Revista Cubana de Higiene y Epidemiología. 2004;42(2):5-12.

9. Romero M, Suárez I, Fajardo J, Barón B. Sífilis maligna en el paciente infectado por el virus de la inmunodeficiencia humana (VIH): descripción de un caso y revisión de la literatura. Anales de Medicina Interna. 2003;20(7):45-48.

10. Picconi A. Detección De Virus Papiloma Humano En La Prevención Del Cáncer Cérvico-Uterino. Medicina (Buenos Aires). 2013;73:585-596

11. Bouvard V, Baan $R$, Straif $K$, et al. A review of human carcinogens - Part B: biological agents. Lancet Oncol. 2009;10:321-322.

12. Bruni L, Diaz M, Castellsagué X, Ferrer E, Bosch FX, de SanJosé S. Cervical human papillomavirus prevalence in 5 continents: meta-analysis of 1 million women with normal cytological findings. J Infect Dis. 2010; 202(12):1789-1799.

13. Cuzick J, Bergeron C, Von Knebel D, et al. New technologies and procedures for cervical cancer screening. Vaccine. 2012;30(S5):107-116

14. Martínez A, Baldiris R, Díaz A. Papiloma bucal producido por VPH y su relación con carcinoma. Rev. Clín. Med. Fam. 2012;5(2):144-145.

15. Elizondo A, Del Pino G, Salgado M. Hiperplasia epitelial focal: Actualidades y tratamiento. Revista Mexicana de Cirugía Bucal y Maxilofacial. 2010;6(3):111-115.

16. Aguayo I, Moreno C, Vano S, Jaen P. Chancro sifilítico en el labio: una localización poco frecuente. Cartas cientificas / Enferm Infecc Microbiol Clin. 2010; 28(2):134-140

17. Pinilla G, Chavarro B, Moreno N, Navarrete J, Muñoz L. Determinación de los genes, 16S ADNr, polA, y TpN47, en la detección de Treponema pallidum subsp. pallidum para el diagnóstico de sífilis congénita. Nova. 2015; 13 ( 24 ): 17-25. 
18. Quattordio L, Milani P, Milani H. Serological diagnosis of syphilis: correlation of results according to available techniques in the laboratory. Acta bioquím. clín. latinoam. 2004;38(3):301-306.

19. Stone D, Moheng M, Rolih S, Sinor L. Capture-S, a non treponemal solid-phase erythrocyte adherence assay for serological detection of syphilis. J Clin Microbiol. 1997;35(1):217-222

20. Castro S, Guzmán D. Manifestaciones bucales por sífilis secundaria. Reporte de un caso clínico. Rev. Cient. Odontol. 2015;11(1):20-25

21. Chairez P, Vega M, Zambrano G, García A, Araceli I, Cuevas J. Presence of Human Papillomavirus in Oral Cavity: Review and Update of Literature. Int. J. Odontostomat. 2015;9(2):233-238.

22. Delgado E, Sandoval M, Guzmán J, Valdivia A. Condiloma viral en lengua: presentación de un caso clínico y revisión bibliográfica. Archivos en Medicina Familiar. 2011;13(1):74-76

23. Cohen MS. When people with HIV get syphilis: triple jeopardy. Sex Transm Dis. 2006; 149-150

24. Díaz-Franco A, Noguer-Zambrano I, Cano-Portero R. Vigilancia epidemiológica de las infecciones de transmisión sexual. España, 1995-2003. Med Clin. 2005; 125(14):529-530.

25. Muñoz M, Rodríguez A, Camacho F. Sexually transmitted diseases in 1161 HIV-positive patients: a 38-month prospective study in southern Spain. J Eur Acad Dermatol Venereol. 1998;11(3):221-6.

26. Vall-Mayans M, Casals M, Vives A, Loureiro E, Armengol P, Sanz B. Reemergencia de la sífilis infecciosa en varones homosexuales y coinfección por el virus de la inmunodeficiencia humana en Barcelona, 2002-2003. Med Clin. 2006;126(3):94-96.

27. Marra C, Tantalo L, Maxwell C, Dougherty K, Wood B. Alternative cerobrospinal fluid tests to diagnose neurosyphilis in HIV-infected individuals. Neurology. 2004;63(1):85-88.

28. Tellez L, Vielma S, Saberio A, Mendoza J, Pérez L, Castillo P, Noguera M. Relación de la infección por VIH en pacientes con lesiones preinvasoras de cérvix asociadas a VPH. Revista Médica de la Extensión Portuguesa. 2007;1(1):5-12. 